\title{
Printers Point
}

National Cancer Institute

\section{Source}

National Cancer Institute. Printers Point. NCI Thesaurus. Code C71237.

A unit of leng th equal to 0.013837 inch or 0.35145 millimeter. 